\title{
Relationships between type of pain and work participation in people with long-standing spinal cord injury: results from a cross-sectional study
}

\author{
Ellen H. Roels ${ }^{1}$ - Michiel F. Reneman ${ }^{1}$ - Janneke Stolwijk-Swuste ${ }^{2} \cdot$ Charlotte C. van Laake-Geelen $^{3}$. \\ Sonja de $\operatorname{Groot}^{4,5} \cdot$ Jacinthe J.E. Adriaansen ${ }^{2}$ Marcel W.M. Post ${ }^{1,2}$
}

Received: 13 September 2017 / Revised: 29 November 2017 / Accepted: 1 December 2017 / Published online: 11 January 2018

(c) International Spinal Cord Society 2018

\begin{abstract}
Study design Multicentre, cross-sectional study.

Objectives To describe the relationships between the presence of (different types of) pain and participation in paid work in people with long-standing spinal cord injury (SCI). Furthermore, the associations of pain-related work limitations, age, gender, relationship, education, lesion level, and time since injury (TSI) with work participation (WP) were investigated.

Setting The Netherlands.

Methods Individuals $(n=265)$ with SCI for $\geq 10$ years were included. Data were collected through a structured consultation with a rehabilitation physician and self-report questionnaire. Descriptive statistics and logistic regression analysis were performed.

Results Median age of participants was 47.9 years, median time since injury was 22 years, $73 \%$ were male, $69 \%$ had complete SCI and 59\% had paraplegia, 50\% had paid work, $63 \%$ reported musculoskeletal pain, $49 \%$ reported neuropathic pain, and $31 \%$ reported other pain. Self-reported pain-related work limitations were significantly $(V=0.26$ and $V=0.27)$ related to WP. In bivariable logistic regression analyses, no statistically significant relationships between type of pain and WP were observed. Younger age $(\mathrm{OR}=0.96)$, male gender $(\mathrm{OR}=0.52)$, a stable relationship $(\mathrm{OR}=1.70)$, and shorter time since SCI $(\mathrm{OR}=0.97)$ were significantly associated with a higher chance of being employed. Multivariable analysis confirmed these findings and in addition showed a higher level of education to be positively related with WP.

Conclusion Age, gender, relationship, education, TSI and self-reported work limitations showed a relationship with WP. Different types of pain were unrelated to WP.

Sponsorship Fonds NutsOHRA through the Dutch Organization for Health Research and Development (ZonMw), Project number 89000006.
\end{abstract}

\section{Introduction}

Spinal cord injury (SCI) has a major impact on all domains of life, including work participation (WP). In case of SCI, WP is related to greater life satisfaction, higher level of activities, and better overall health [1]. WP is further associated with longevity in persons with SCI [2]. Beside the benefits of WP for people with SCI, a high WP is also beneficial to society [3]. WP of people with SCI, however, is low compared to the general population; work rates following SCI reported in the
Ellen H. Roels

e.h.roels@umcg.nl

1 University of Groningen, University Medical Center Groningen, Department of Rehabilitation Medicine, Center for Rehabilitation, Groningen, The Netherlands

2 Center of Excellence for Rehabilitation Medicine, Brain Center Rudolf Magnus, University Medical Center Utrecht, Utrecht University and De Hoogstraat Rehabilitation, Utrecht The Netherlands
3 Adelante Centre of Expertise in Rehabilitation and Audiology, Hoensbroek, The Netherlands

4 University of Groningen, Reade, Amsterdam The Netherlands

5 Amsterdam Rehabilitation Research Institute, University Medical Center Groningen, Center for Human Movement Sciences, Groningen, The Netherlands 
literature vary from 3 to $80 \%$ [4-6]. In studies performed in the Netherlands, WP rates of $37-67 \%$ have been reported (http://www.dehoogstraat.nl/images/products/800/eindra pportilias-definitief.pdf) [7-9], compared to $75 \%$ of the general Dutch population (Eurostat-tradingeconomics.com). Among factors that contribute to WP in people with SCI are non-modifiable factors such as age and gender, and it has been recommended that studies should focus on associations between modifiable factors and WP [10].

Pain might be such a modifiable factor and treating pain adequately might improve WP outcomes. The prevalence of pain in SCI is reported to be 67-85\%, varying with type of pain and time since injury (TSI) [11-15]. In one study, onethird of the participants with SCI reported extreme or excruciating pain [14]. WP and pain relief have been described as within the three most unmet needs in one European SCI sample [16]. Despite the high prevalence of pain, most studies on pain and WP address pain as a general condition without distinguishing different types of pain. The association between uncategorized pain in SCI and WP has been examined in several studies; pain was negatively related to WP [17, 18] and employment [19]. Pain was described as a barrier for social and community participation in SCI [20]. Furthermore, people with mild pain were almost three times more likely to be employed compared to those with severe pain [21]. Pain also moderated the associations between age and age at injury and WP; in participants of 50 years and older but not younger participants, pain was associated with a lower likelihood of WP [22]. Only few studies address the relationship between a specific pain type mainly neuropathic pain and WP; among people with SCI and neuropathic pain, the proportion of employed people was significantly lower if the pain was more severe [23] and the presence of neuropathic pain decreased WP among men with SCI, but not in women [24]. In contrast, another study found shoulder pain at discharge from rehabilitation not to be associated with limitations in activities and participation 5 years later [25]. Also, in a qualitative study, WP was considered distractive from pain [26], suggesting that pain not necessarily negatively impacts WP.

Vocational rehabilitation providers need to be aware of chronic pain issues and discuss this with their clients [27]. Because of differences in treatment approaches, it seems clinically relevant to know which type of pain is potentially limiting for WP in people with SCI, however literature on this specific topic is lacking. Having more insights in this relationship, might be useful to guide rehabilitation.

To our knowledge, no studies have been conducted that aimed to objectify the relationship between different types of pain simultaneously and WP in people with SCI.

The aim of the study was to analyze the relationships between different types of pain and WP in people with SCI.
Secondly, we aimed to evaluate the relationships between self-reported pain-related limitations and actual WP, hypothesizing an inverse relationship between pain-related limitations and WP. Furthermore, the relationships between age, gender, relationship, level of education, lesion level, TSI and WP were investigated.

\section{Methods}

\section{Design}

This study is part of the Dutch multicentre research program "Active Lifestyle Rehabilitation Interventions in aging Spinal Cord injury (ALLRISC)", a TSI stratified, crosssectional study amongst persons with long-standing SCI living in the Netherlands [28]. The research protocol was approved by the Medical Ethics Committee of the University Medical Centre Utrecht (METC protocol number: 11/156). We certify that all applicable institutional and governmental regulations concerning the ethical use of human volunteers were followed during the course of this research. All participants gave written informed consent.

\section{Participants}

Inclusion criteria were traumatic and non-traumatic SCI with a TSI $\geq 10$ years, age at injury between 18 and 35 years, age at the time of the study between 28 and 65 years, and wheelchair-user for longer distances $(>500 \mathrm{~m})$. The exclusion criteria were having SCI due to a malignant tumor and insufficient knowledge of the Dutch language to respond to an oral interview or to understand test instructions.

\section{Procedure}

Eligible participants were identified in databases from all eight Dutch rehabilitation centers specialized in SCI rehabilitation. As the aim was to include 30-35 persons per center and a response rate of $\sim 50 \%$ was expected, 62 persons per center were invited to join the study. If the number of eligible persons allowed it, a random sample was drawn at each center. If the response was below $n=30$, an additional sample was drawn at that center. The participants had a one day visit to the rehabilitation center for a check-up, consisting of an extensive medical assessment and physical examination including an American Spinal Injury Association (ASIA) examination performed by a SCI rehabilitation physician. Two weeks before the visit to the rehabilitation center, participants were asked to complete a self-report questionnaire. Information on pain was obtained from the medical assessment and information on WP from the questionnaire. 


\section{Instruments}

Participant characteristics such as age at time of measurement, gender, relationship status (in a stable relationship or married versus single), educational level (high: university or high level secondary school or low: secondary school, primary school, or no education), SCI level, and ASIA Impairment Scale (AIS; classified as complete AIS A versus incomplete AIS B, C, or D) according to the International Standards for Neurological Classification of SCI [29] and TSI were collected.

WP status was classified as employed, disability pension, retired, housekeeping work, volunteer work, study/education, or other (combinations possible). Hours of WP per week were classified into six categories: no paid work, $1-8$ $\mathrm{h}, 9-16 \mathrm{~h}, 17-24 \mathrm{~h}, 25-35 \mathrm{~h}$, and more than $35 \mathrm{~h}$ of paid work per week. To predict the likelihood of WP, we rearranged the above categories in non-workers $(0 \mathrm{~h}$ per week) and workers ( $\geq 1 \mathrm{~h}$ per week) based on the guidelines of the International Labour Organisation (https://www.cbs. nl/en-gb/news/2014/27/statistics-netherlands-opts-forinternational-definitions-of-unemployment-and-inflation).

Pain during the last 3 months was assessed. If the participant reported to have pain, standardized questions on the type of pain were completed. Type of pain was classified based on the classification system developed by the SCI Pain Task Force of the International Association for the Study of Pain [30]. Pain was then reported by the rehabilitation physician as musculoskeletal pain (above or at SCI level), visceral pain, pain as a consequence of spasticity, neuropathic pain (above, at or below SCI level), pain as a consequence of syrinx, other pain (as a consequence of SCI or not), or no pain. When patients reported other pain, they were asked to describe their type of pain. Descriptions of other pain were re-classified if possible, e.g., shoulder pain was classified as musculoskeletal pain if above or at SCI level; pain with neuropathic characteristics (carpal tunnel syndrome, painful hyperesthesia at injury level, tingling pain in legs, and pinning feeling in legs) was classified as neuropathic pain and abdominal pain was classified as visceral pain. Types of pain were then merged into three main categories: musculoskeletal pain (above or at SCI level), neuropathic pain (above, at or below SCI level) including pain as a consequence of syrinx, and other pain including pain as a consequence of spasticity and visceral pain.

Furthermore participants were asked to rate the influence of musculoskeletal and neuropathic pain on their capacity to participate in vocational and household activities. This was scored from 0 (no limitation in participation due to pain) to 10 (full limitation due to pain). Responses were categorized in three groups for further analysis: no pain-related limitation (score 0 ), moderate pain-related limitation (score 1 to 4), and severe pain-related limitation (score 5 to 10 ).

\section{Statistical analysis}

If specific data were missing, this was mentioned in the frequency tables. Participants with missing information on pain or WP variables were excluded from the analyses. Descriptive analyses were performed to describe demographic, injury-related characteristics, pain, and WP characteristics. Baseline characteristics are expressed in medians (interquartile range) or $n(\%)$. To assess the association of WP and type of pain, bivariable, and multivariable analyses were performed. In the bivariable logistic regression analyses WP (working 0 versus $\geq 1 \mathrm{~h}$ per week) was the dependent variable and independent variables were age, gender, stable relationship, education level, lesion level, TSI, musculoskeletal pain, neuropathic pain, and other pain. Multivariable logistic regression analyses were used to predict WP for all pain types (musculoskeletal pain, neuropathic pain, other pain, and including all potential confounders at once: age at time of measurement, gender, stable relationship, education level, lesion level, and TSI). Before performing the multivariable logistic regression, variables were checked for multicollinearity by calculating the Pearson's correlation coefficient and the variance inflation factor (VIF). As a high Pearson's correlation coefficient $(r=0.864, \mathrm{p}=0.01)$ between TSI and age and a high VIF (4.08 for age and 4.09 for TSI) was observed, the multivariable analysis was performed twice, with either age or TSI excluded from the model. Odds ratios (OR) with $95 \%$ confidence intervals (CI) were reported.

Associations between WP and experienced limitations in participation in vocational and household activities due to musculoskeletal or neuropathic pain were analyzed with Cramers' $V$ test. The $V$-value was interpreted as follows: 0.1: small effect, 0.3: medium effect, and 0.5: large effect [31].

Data were analyzed using the Statistical Package for Social Sciences for Windows, version 23.0 (IBM Corp., Armonk, NY). Results were considered statistically significant when $p<0.05$.

\section{Results}

From all 282 participants in the ALLRISC study, information on pain and WP was available of 265 participants and those were selected for the current study. Demographic, injuryrelated, and WP characteristics are described in Table 1.

Table 2 shows the characteristics of the different types of pain. Musculoskeletal pain was the most frequently occurring pain, followed by neuropathic pain and other pain. The table also shows the incidence of experienced pain-related limitations on capacity to participate in vocational/household activities. 
Table 1 Demographic, injuryrelated, and WP characteristics of the study sample $(n=265)$

\begin{tabular}{ll}
\hline & Median (IQR) or $n(\%)$ \\
\hline Age (years) & $47.9(41.7-55)$ \\
Sex (\% of male) & $194(73 \%)$ \\
Relationship (\% of & $162(61 \%)$ \\
stable relationship) & \\
Education & \\
High level & $113(43 \%)$ \\
Low level & $146(55 \%)$ \\
Missing & $6(2 \%)$ \\
Cause (traumatic) & $240(91 \%)$ \\
SCI level (paraplegia) & $157(59 \%)$ \\
Missing & $1(0.4 \%)$ \\
ASIA Impairment Scale & \\
A & $182(69 \%)$ \\
B & $34(13 \%)$ \\
C & $25(9 \%)$ \\
D & $23(9 \%)$ \\
Missing & $1(0.4 \%)$ \\
Time since injury & $22(17-31)$ \\
(years) & $148(56 \%)$ \\
Disability pension & $6(2 \%)$ \\
Retired & $27(10 \%)$ \\
Housekeeping work & $39(15 \%)$ \\
Volunteer work & $5(2 \%)$ \\
Htudy/education & $17(6 \%)$ \\
range & $23(9 \%)$ \\
Hours paid employment & \\
1-8 h h per per week & $133(50 \%)$ \\
25-35 h per week &
\end{tabular}

Table 3 describes the relationships between pain-related restrictions to participate in vocational/household activities and WP. Significant relationships (medium effect) between more restrictions due to musculoskeletal pain (Cramer's $V$ $=0.26 ; p=0.004)$ and more restrictions due to neuropathic pain (Cramer's $V=0.27 ; \mathrm{p}=0.016$ ) with lower WP were observed.

Table 4 shows the results for the logistic regression analyses. Analyses indicated that the relationship between type of pain and WP is non-significant, both in the bivariable analyses and in the multivariable model. In bivariable analyses, younger age at time of measurement, male gender, and having a stable relationship were associated with a higher chance of being employed. A longer TSI was associated with a lower chance of employment. Multivariable analysis confirmed the above findings and showed furthermore that having a higher education was associated with a higher chance of being employed (Nagelkerke $R^{2}$ including TSI $=0.115$; Nagelkerke $R^{2}$ including age $=0.141$ ).

\section{Discussion}

To the best of our knowledge, the present study is the first to analyze the association between different types of pain and WP among people with long- standing SCI. We expected to find a relationship between different types of pain and WP in SCI, however, no relationships were observed. This could be explained by several reasons. Literature has shown a relationship between other medical complications and WP 
Table 2 Characteristics of pain and pain-related limitations in the study sample $(n=265)$

\begin{tabular}{ll}
\hline & $n(\%)$ \\
\hline MSKP above or at injury & $168(63 \%)$ \\
NP & $130(49 \%)$ \\
Above level & $14(5 \%)$ \\
Missing & $1(0.4 \%)$ \\
At level & $35(13 \%)$ \\
Below level & $102(39 \%)$ \\
As consequence of syrinx & $6(2 \%)$ \\
Other pain & $81(31 \%)$ \\
As consequence of spasticity & $27(10 \%)$ \\
Visceral pain & $41(16 \%)$ \\
Other SCI-related pain & $14(5 \%)$ \\
Other non SCI-related pain & $11(4 \%)$ \\
No pain & $48(18 \%)$ \\
Self-reported MSKP-related limitations on WP & \\
No limitation & $79(30 \%)$ \\
Moderate limitation & $50(19 \%)$ \\
Severe limitation & $36(14 \%)$ \\
Self-reported NP-related limitations on WP & \\
No limitation & $69(26 \%)$ \\
Moderate limitation & $22(8 \%)$ \\
Severe limitation & $26(10 \%)$ \\
\hline$M S K P$ musculoskeletal pain, NP neuropathic & \\
participation & \\
&
\end{tabular}

in SCI [6]. These factors were not taken into account in our study and potentially have an impact. Furthermore pain was evaluated over the last 3 months, whereas other studies reporting an association between pain and WP have investigated the effect of pain as being present over a longer period of time [23, 24]. WP status therefore might have been decided upon much earlier. Also, information on pain intensity was limited available and therefore the severity of pain could not be taken into account in our study whereas information of intensity of pain was considered in other studies showing an association between pain and WP [18,

Table 3 Associations between experienced pain-related restrictions in participation in vocational/household activities and WP

\begin{tabular}{llllll}
\hline & $\begin{array}{l}\text { Musculoskeletal pain } \\
\text { above/at injury } \\
(n=165)\end{array}$ & & $\begin{array}{l}\text { Neuropathic pain } \\
(n=117)\end{array}$ \\
\cline { 2 - 3 } \cline { 5 - 6 } & $\begin{array}{l}\text { No WP } \\
n(\%)\end{array}$ & WP & & $\begin{array}{l}\text { No WP } \\
n(\%)\end{array}$ & WP \\
& $30(18 \%)$ & $49(30 \%)$ & $30(26 \%)$ & $39(33 \%)$ \\
\hline No limitation & $29(18 \%)$ & $21(13 \%)$ & $8(7 \%)$ & $14(12 \%)$ \\
Moderate limitation & $25(15 \%)$ & $11(7 \%)$ & $19(16 \%)$ & $7(6 \%)$ \\
\hline Severe limitation & 25 & & &
\end{tabular}

$h$ hours, WP work participation
19, 21, 23]. Interestingly, however, another study has shown incongruence between pain intensity and interference in WP in people with SCI as persons with AIS D were reporting less pain intensity and greater pain interference [21]. This implicates that pain interference, and not pain severity alone, has to be considered for treatment with the aim of optimal functioning [21].

In addition, specifically for SCI the exact mechanisms of chronic pain have not clearly been described, but it is known that the impact of chronic pain in SCI is associated with different coping strategies [32], which might influence the participation level. In addition, a previous study on the same study group, showed surprisingly weak associations between secondary health conditions (SHC), such as musculoskeletal pain, and quality of life. The authors assumed that with a median of four SHC over the last 3 months, participants with SCI do experience SHC regularly and have adapted to the presence of SHC. Therefore, SHC potentially no longer influence the impact of the SHC on quality of life [28]. A similar finding could possibly explain the lack of a relationship between pain and WP. Also, as known from other diagnostic groups, chronic pain (>3 months present) leads to changes on a sensory, muscular, autonomic, and psychosocial dimension and there is a discrepancy of structural damage, pain sensation, and pain 
Table 4 Logistic regression analysis with WP as dependent variable

\begin{tabular}{|c|c|c|c|c|c|c|c|c|c|c|}
\hline & \multicolumn{4}{|c|}{ Bivariable analysis } & \multicolumn{6}{|c|}{ Multivariable analysis $(n=258)$} \\
\hline & \multirow[b]{2}{*}{ Odds ratio } & \multirow[b]{2}{*}{$95 \% \mathrm{CI}$} & \multirow[b]{2}{*}{$p$} & \multirow[b]{2}{*}{$n$} & \multicolumn{3}{|c|}{ Including TSI } & \multicolumn{3}{|c|}{ Including age } \\
\hline & & & & & Odds ratio & $95 \% \mathrm{CI}$ & $p$ & Odds ratio & $95 \% \mathrm{CI}$ & $p$ \\
\hline Age & 0.958 & $0.931-0.985$ & 0.003 & 265 & - & - & - & 0.953 & $0.924-0.982$ & 0.002 \\
\hline Gender & 0.520 & $0.298-0.907$ & 0.021 & 265 & 0.426 & $0.230-0.788$ & 0.007 & 0.408 & $0.219-0.763$ & 0.005 \\
\hline Relationship & 1.701 & $1.033-2.802$ & 0.037 & 265 & 1.728 & $1.016-2.938$ & 0.043 & 1.711 & $1.001-2.924$ & 0.050 \\
\hline Education level & 1.540 & $0.939-2.525$ & 0.087 & 259 & 1.785 & $1.047-3.044$ & 0.033 & 1.733 & $1.010-2.973$ & 0.046 \\
\hline Lesion level & 1.328 & $0.811-2.173$ & 0.260 & 264 & 1.476 & $0.865-2.518$ & 0.153 & 1.567 & $0.912-2.694$ & 0.104 \\
\hline Time since injury & 0.972 & $0.945-0.998$ & 0.037 & 265 & 0.969 & $0.941-0.997$ & 0.030 & - & - & - \\
\hline Musculoskeletal pain & 0.840 & $0.509-1.385$ & 0.494 & 265 & 0.968 & $0.562-1.668$ & 0.907 & 0.981 & $0.567-1.695$ & 0.944 \\
\hline Neuropathic pain & 1.078 & $0.666-1.745$ & 0.760 & 265 & 1.108 & $0.658-1.865$ & 0.700 & 1.141 & $0.674-1.933$ & 0.623 \\
\hline Other pain & 1.394 & $0.824-2.356$ & 0.215 & 265 & 1.356 & $0.772-2.382$ & 0.290 & 1.4 & $0.793-2.470$ & 0.246 \\
\hline
\end{tabular}

CI confidence interval, TSI time since injury

behavior [33]. In chronic multifactorial pain, e.g., different domains of the biopsychosocial model (fear avoidance, pain catastrophizing, perceived workload, pain acceptance, life control, and pain self-efficacy) have been shown to influence "staying at work" [34]. Similar findings have been shown in persons with physical disabilities, underlining the importance of psychosocial factors as predictors of pain and functioning [35]. Specifically for SCI, psychological factors, particularly beliefs about pain (including catastrophizing) and pain-related coping strategies (including passive coping), are predictors of pain outcomes [36]. Therefore, the beliefs about pain and the pain-related coping strategy may contribute to the relationship between pain and WP. This could partially be confirmed in our study as the self-reported pain-related work limitation was associated with WP assuming a relationship between pain coping/ behavior and WP. Depending on the coping mechanism, people with SCI might experience pain differently leading to different pain responses and pain interference independent from type of pain and resulting in different participation outcomes. Clinicians should therefore not entirely focus on treating pain presence as such, but also the mechanism of coping with pain and the pain behavior should be analyzed and optimized requiring a biopsycosocial approach which goes beyond a strictly medical approach only that is now often applied in clinical settings. In addition, medication to treat pain often has significant side effects in cognitive functioning leading to another barrier for WP. This could be an extra motivation for switching towards a biopsychosocial approach on treating pain in SCI. This biopsychosocial approach is already being used in pain rehabilitation programs [33], however is not standardly introduced in SCI rehabilitation programs.

As expected, an association was seen between painrelated work and household limitations and WP. This shows that our participants did experience vocational limitations due to pain, despite the lack of such an association in the other statistical analyses. Literature on this specific association is lacking, and this discrepancy warrants further research.

The present study furthermore provides useful information on non-pain-related influencing factors and WP in SCI. The WP rate in our study is high comparing to international studies and is in line with a relatively recent Dutch study with similar inclusion criteria showing return to work rates of $51 \%$ for $\geq 1 \mathrm{~h}$ per week and 42.6 for $\geq 12 \mathrm{~h}$ per week at 5 years following discharge from the inpatient rehabilitation [9]. A previous national study showed rates of $37 \%$ using a cross-sectional design and including participants with a shorter post-injury duration [8]. Another Dutch study showed $60 \%$ of individuals with SCI work $>4 \mathrm{~h}$ per week (mean TSI 84 months with standard deviation 29), however, this latest study involved a small number of participants $(n=57)$ [7]. We found similar pain prevalence percentages as previously reported for musculoskeletal pain (59\% experiencing pain at 5 years following injury versus $63 \%$ in our study) and below level neuropathic pain (34 versus 39\% in our study), however the percentages for at level neuropathic pain previously reported differed (41 versus $13 \%$ in our study) [14]. Our study furthermore confirms that a lower age, being male, a stable relationship, a higher level of education, and a shorter TSI enhance the chances of WP. This is consistent with previous studies indicating that many other, mostly non-modifiable, factors are related to WP [8, $37,38]$.

\section{Limitations}

From the many variables measured in the ALLRISC study, we have a priory chosen the variables that we considered 
most likely to impact the association between pain and work: age, sex, relationship, education, lesion level, TSI, and type of pain. Many other health related variables such as mental health, bladder, bowel, and skin issues but also demographic variables such as income were not considered for analysis. Those variables might have influenced the relationship between type of pain and WP, but as we did not observe such an association in the bivariable and multivariable analyses, it is unlikely that the inclusion of more or other determinants would have changed our results.

Second, the participants in this study do not represent the full SCI community: participants had to be wheelchair-user for longer distances $(>500 \mathrm{~m})$ and injured between 18 and 35 years of age and therefore only few had an incomplete SCI. Also, relatively few non-traumatic SCI were included in the study sample. As the study only included longstanding SCI and no longitudinal follow up had taken place, there is a possibility of longitudinal selection bias. In addition, information on non-responders is missing.

Further, limited information on pain was collected, e.g., intensity of neuropathic pain was not asked for. Also musculoskeletal pain was only asked for if present above or at SCI level excluding a group of patients with musculoskeletal pain below injury level that were now included in the "other" group. Furthermore, as part of the questionnaire, the question on experienced pain-related work limitation also included experienced limitation on domestic work (and thus not only paid work). Therefore, this information was not entirely valid as information on effect of pain on WP.

Also the potential distractive effect of WP on pain was not specifically considered in this study [26].

Future research should focus on relationships between pain intensity, pain interference and WP. To gain comprehensive information on pain and pain interference the International SCI Pain Basic Data Set Version 2.0 could be used as a screening tool for future research on this topic (http://www.iscos.org.uk/sitefiles/2013\%2006\%2011_ International\%20SCI20Pain\%20Basic\%20Data\%20). Furthermore, the impact of pain coping mechanisms and behavior on WP needs further investigation.

Acknowledgements We thank all ALLRISC research assistants and SCI rehabilitation physicians for collecting all the data and the following participating Dutch rehabilitation centers: Rehabilitation Center De Hoogstraat (Utrecht), Reade Center for Rehabilitation (Amsterdam); Rehabilitation Center Het Roessingh (Enschede); Adelante Rehabilitation Center (Hoensbroek); Sint Maartenskliniek (Nijmegen); University Medical Center Groningen, Center for Rehabilitation (Groningen); Rehabilitation Center Heliomare (Wijk aan Zee), and Rijndam Rehabilitation Center (Rotterdam).

Funding ALLRISC is sponsored by "Fonds NutsOHRA" under the responsibility of the Netherlands. Organization for Health Research and Development (ZonMw), Project Number 89000006.

\section{Compliance with ethical standards}

Conflict of interest The authors declare that they have no conflict of interest.

\section{References}

1. Krause JS. Adjustment to life after spinal cord injury: a comparison among three participant groups based on employment status. Rehabil Couns Bull. 1992;35:218-29.

2. Krause JS, Saunders LL, Acuna J. Gainful employment and risk of mortality after spinal cord injury: effects beyond that of demographic, injury and socioeconomic factors. Spinal Cord. 2012;50:784-8.

3. DeVivo MJ. Causes and costs of spinal cord injury in the United States. Spinal Cord. 2013;35:809-13.

4. Young AE, Murphy GC. Employment status after spinal cord injury (1992-2005): a review with implications for interpretation, evaluation, further research, and clinical practice. Int $\mathbf{J}$ Rehabil Res. 2009;32:1-11.

5. Ottomanelli L, Lind L. Review of critical factors related to employment after spinal cord injury: implications for research and vocational services. J Spinal Cord Med. 2009;32:503-31.

6. Lidal IB, Huynh TK, Biering-Sørensen F. Return to work following spinal cord injury: a review. Disabil Rehabil. 2007;29:1341-75.

7. Schönherr MC, Groothoff JW, Mulder GA, Eisma WH. Vocational perspectives after spinal cord injury. Clin Rehabil. 2005;19:200-8.

8. Tomassen PC, Post MW, van Asbeck FW. Return to work after spinal cord injury. Spinal Cord. 2000;38:51-5.

9. Ferdiana A, Post MWM, De Groot S, Bültmann U, Van Der Klink JJL. Predictors of return to work 5 years after discharge for wheelchair-dependent individuals with spinal cord injury. $\mathbf{J}$ Rehabil Med. 2014;46:984-90.

10. Trenaman L, Miller WC, Queree M, Escorpizo R, Team SR. Modifiable and non-modifiable factors associated with employment outcomes following spinal cord injury: a systematic review. J Spinal Cord Med. 2015;38:422-31.

11. Jensen MP, Kuehn CM, Amtmann D, Cardenas DD. Symptom burden in persons with spinal cord injury. Arch Phys Med Rehabil. 2007;88:638-45.

12. Ravenscroft A, Ahmed YS, Burnside IG. Chronic pain after SCI. A patient survey. Spinal Cord J Int Med Soc Paraplegia. 2000;38:611-4.

13. Widerström-Noga EG, Felipe-Cuervo E, Broton JG, Duncan RC, Yezierski RP. Perceived difficulty in dealing with consequences of spinal cord injury. Arch Phys Med Rehabil. 1999;80:580-6.

14. Siddall PJ, McClelland JM, Rutkowski SB, Cousins MJ. A longitudinal study of the prevalence and characteristics of pain in the first 5 years following spinal cord injury. Pain. 2003;103:249-57.

15. Stormer S, Gerner HJ, Gruninger W, Metzmacher K, Follinger S, Wienke C, et al. Chronic pain/dysaesthesiae in spinal cord injury patients: results of a multicentre study. Spinal Cord. 1997;35:446-55.

16. Kennedy P, Lude P, Taylor N. Quality of life, social participation, appraisals and coping post spinal cord injury: a review of four community samples. Spinal Cord. 2006;44:95-105.

17. Anderson CJ, Vogel LC. Employment outcomes of adults who sustained spinal cord injuries as children or adolescents. Arch Phys Med Rehabil. 2002;83:791-801.

18. Jensen MP, Hoffman aJ, Cardenas DD. Chronic pain in individuals with spinal cord injury: a survey and longitudinal study. Spinal Cord. 2005;43:704-12. 
19. Marti A, Reinhardt JD, Graf S, Escorpizo R, Post MWM. To work or not to work: labour market participation of people with spinal cord injury living in Switzerland. Spinal Cord. 2012;50:521-6.

20. Barclay L, McDonald R, Lentin P. Social and community participation following spinal cord injury: a critical review. Int $\mathrm{J}$ Rehabil Res. 2015;38:1-19.

21. Forchheimer MB, Richards JS, Chiodo AE, Bryce TN, DydsonHudson T. Cut point determination in the measurement of pain and its relationship to psychosocial and functional measures after traumatic spinal cord injury: a retrospective model spinal cord injury system analysis. Arch Phys Med Rehabil. 2011;92:419-24.

22. Marti A, Boes S, Lay V, Escorpizo R, Trezzini B. The association between chronological age, age at injury and employment: is there a mediating effect of secondary health conditions? Spinal Cord. 2016;54:239-44.

23. Mann R, Schaefer C, Sadosky A, Bergstrom F, Baik R, Parsons B, et al. Burden of spinal cord injury-related neuropathic pain in the United States: retrospective chart review and cross-sectional survey. Spinal Cord. 2013;51:564-70.

24. Valtonen K, Karlsson AK, Alaranta H, Viikari-Juntura E. Work participation among persons with traumatic spinal cord injury and meningomyelocele1. J Rehabil Med. 2006;38:192-200.

25. Eriks-Hoogland I, De Groot S, Snoek G, Stucki G, Post M, Van Der Woude L. Association of shoulder problems in persons with spinal cord injury at discharge from inpatient rehabilitation with activities and participation 5 years later. Arch Phys Med Rehabil. 2016;97:84-91.

26. Leiulfsrud AS, Ruoranen K, Ostermann A, Reinhardt JD. The meaning of employment from the perspective of persons with spinal cord injuries in six European countries. Work. 2016;55:133-44.

27 Schultz IZ, Gatchel RJ. Handbook of return to work: from research to practice. New York, NY: Springer; 2016.

28. Adriaansen JJE, van Asbeck FWA, Lindeman E, van der Woude LHV, de Groot S, Post MWM. Secondary health conditions in persons with a spinal cord injury for at least 10 years: design of a comprehensive long-term cross-sectional study. Disabil Rehabil. 2013;35:1104-10.

29. Kirshblum SC, Waring W, Biering-Sorensen F, Burns SP, Johansen M, Schmidt-Read M, et al. Reference for the 2011 revision of the international standards for neurological classification of spinal cord injury. J Spinal Cord Med. 2011;34: 547-54.

30. Burchiel KJ, Hsu FP. Pain and spasticity after spinal cord injury: mechanisms and treatment. Spine. 2001;26:S146-S60.

31 Cohen J. Statistical power analysis for the behavioral sciences. 2nd ed. Hillsdale, NJ: Lawrence Erlbaum;1988.

32. Wollaars MM, Post MWM, van Asbeck FWA, Brand N. Spinal cord injury pain: the influence of psychologic factors and impact on quality of life. Clin J Pain. 2007;23:383-91.

33 Devulder JER, Smeets RP. Revalidatie voor Volwassenen. Assen: Koninklijke Van Gorcum; 2014. pp 271-87.

34. De Vries HJ, Reneman MF, Groothoff JW, Geertzen JHB, Brouwer S. Workers who stay at work despite chronic nonspecific musculoskeletal pain: do they differ from workers with sick leave? J Occup Rehabil. 2012;22:489-502.

35. Jensen MP, Moore MR, Bockow TB, Ehde DM, Engel JM. Psychosocial factors and adjustment to chronic pain in persons with physical disabilities: a systematic review. Arch Phys Med Rehabil. 2011;92:146-60.

36. Molton IR, Stoelb BL, Jensen MP, Ehde DM, Raichle KA, Cardenas DD. Psychosocial factors and adjustment to chronic pain in spinal cord injury: replication and cross-validation. J Rehabil Res Dev. 2009;46:31-42.

37. Hirsh AT, Molton IR, Johnson KL, Bombardier CH, Jensen MP. The relationship of chronological age, age at injury, and duration of injury to employment status in individuals with spinal cord injury. Psychol Inj Law. 2009;2:263-75.

38. Franceschini M, Pagliacci MC, Russo T, Felzani G, Aito S, Marini C. Occurrence and predictors of employment after traumatic spinal cord injury: the GISEM Study. Spinal Cord. 2012;50:238-42. 